\title{
Decay of an unstable state in the presence of multiplicative noise
}

\author{
F. de Pasquale \\ Dipartimento di Fisica, Università degli Studi di Roma "La Sapienza," piazzale Aldo Moro 2, I-00185 Roma, Italy \\ J. M. Sancho and M. San Miguel \\ Departamento de Física Teórica, Universidad de Barcelona, Diagonal 647, 08028 Barcelona, Spain \\ P. Tartaglia \\ Dipartimento di Fisica, Università degli Studi di Roma "La Sapienza," piazzale Aldo Moro 2, I-00185 Roma, Italy
}

(Received 1 August 1985)

\begin{abstract}
The phenomenon of anomalous fluctuations associated with the decay of an unstable state is analyzed in the presence of multiplicative noise. A theory is presented and compared with a numerical simulation. Our results allow us to distinguish the roles of additive and multiplicative noise in the nonlinear relaxation process. We suggest the use of experiments on transient dynamics to understand the effect of these two sources of noise in problems in which parametric noise is thought to be important, such as dye lasers.
\end{abstract}

\section{INTRODUCTION}

The decay of unstable states is one of the fundamental problems in the study of the dynamics of nonequilibrium phenomena and nonlinear relaxation processes. ${ }^{1}$ A new interesting aspect of this problem appears when fluctuations of control parameters are considered. In this paper we suggest that the study of the decay process can be useful to separate the effect of these fluctuations from that of the usual internal noise. A characteristic feature associated with the decay process is the existence of transient anomalous fluctuations. This phenomenon is by now well established and there is broad experimental evidence for it, mostly in studies of transient dynamics of lasers. ${ }^{2}$ The basic theoretical idea used to describe the decay process is that initial fluctuations needed to start the process are amplified in the deterministic evolution. Such amplification causes the anomalous fluctuation phenomenon. This is followed by a saturation regime due to nonlinearities in which fluctuations become again important. These ideas have been developed in several ways. One of them is the quasideterministic theory ${ }^{3}$ (QDT) in which the real process is approximated by the deterministic nonlinear mapping acting on the initial fluctuations.

The usual analysis of the decay processes briefly reviewed above is based on stochastic models of the Langevin type in which fluctuations are modeled by a random force that is independent of the state of the systems (additive noise). It is also of interest to consider fluctuations which depend on the instantaneous state of the system (multiplicative noise). ${ }^{4-8}$ The natural question then arises of the role played by multiplicative noise in the decay of an unstable state. Multiplicative noise arises in microscopic derivations of stochastic models, ${ }^{9}$ but most recent studies of multiplicative stochastic processes are motivated by the consideration of parametric or external noise, that is, fluctuations in the control parameter of nonequilibrium systems. Such parametric fluctuations are known to modify instability thresholds, stationary distributions, and dynamical properties, ${ }^{5-8}$ but the question of the different roles of additive and multiplicative noise in the decay of unstable states has not yet been addressed.

Multiplicative noise of external origin is in general much stronger than additive noise of internal origin. On these grounds, additive noise is often neglected in studies of multiplicative stochastic processes. However, additive weak noise has important consequences in many circumstances. One of them is the situation in which the external noise vanishes at the unstable state. The decay of such a state is only possible due to additive noise. If the unstable state separates into two symmetric stable states, multiplicative noise of this sort breaks the symmetry of the problem confining the system to a subspace of phase space. The symmetry is only restored by additive noise. This symmetry restoring occurs for long times through escape processes from one stable state to the other. The influence of multiplicative noise in the escape process has been discussed in Ref. 10. The purpose of this paper is to analyze the early-time dynamics associated with the decay of the unstable state and to describe the effect of multiplicative noise in this regime, in particular in the phenomenon of anomalous fluctuations. A well-defined physical situation which motivates our analysis is the transient dynamics of a dye laser. Indeed, the anomalous statistical properties of dye lasers have been attributed to fluctuations of the pump parameter. This leads to a model with multiplicative noise analyzed by several authors. $^{11}$ However, it has also been suggested ${ }^{12}$ that in a proper description of this problem additive noise associated with spontaneous emission fluctuations cannot be neglected. This has only been discussed for steady-state properties. An experiment on the transient dynamics of this system and the discussion of the experimental results along the lines of the theoretical analysis presented here should be useful to distinguish the effects of spontaneous emission fluctuations and pumping multiplicative noise in 
the radiation emitted by a dye laser.

The theory of nonlinear relaxation of a system in the presence of additive and multiplicative noise appears to be quite difficult. The main difficulty is, in our opinion, that even in the case in which only multiplicative noise is present, the exact theory for the decay of a state close to an unstable one cannot be simplified in a meaningful way by the methods which have ordinarily been employed in the case of additive noise to deal directly with average quantities. The introduction, for instance, of a linear process in the presence of a self-consistent time-dependent restoring force gives in such a case spurious results for high-order moments. To avoid this, we try to approximate the process itself by exploiting the circumstance when additive noise is expected to be important only close to the unstable state, where multiplicative noise is ineffective. We will see that the QDT developed to study the decay of an unstable state in the case when only additive noise is present can be easily generalized to the present case. QDT gives an approximation for the process itself which is here compared with a direct simulation. We find quite good quantitative agreement as long as the strength of the multiplicative noise is relatively small. The general picture that arises is valid for any value of such strength. The main application that we give is to show how the well-known phenomenon of anomalous fluctuations is modified in the presence of multiplicative noise. We show that the fluctuation enhancement is qualitatively unaffected by multiplicative noise. This fact directly suggests the use of transient statistics experiments to separate the roles of additive and multiplicative noise.

The outline of the paper is as follows. In Sec. II we present the results of a numerical simulation for a prototype model. These results make clear the different role of two sources of noise. In Sec. III we extend the QDT to the case in which multiplicative noise is present. The results of the extended QDT are compared with the simulation. Section IV contains some final remarks on the validity of QDT.

\section{MODEL AND RESULTS OF SIMULATION}

The stochastic process that we consider is defined by the following stochastic differential equation: ${ }^{13}$

$$
\partial_{t} x=r x-x^{3}+\sqrt{2 D} x \eta(t)+\sqrt{2 \epsilon} \xi(t)
$$

where $\eta(t)$ and $\xi(t)$ are two types of uncorrelated Gaussian white noise of zero mean and correlations

$$
\left\langle\eta(t) \eta\left(t^{\prime}\right)\right\rangle=\left\langle\xi(t) \xi\left(t^{\prime}\right)\right\rangle=\delta\left(t-t^{\prime}\right) .
$$

The parameters $D$ and $\epsilon$ measure the strength of the multiplicative noise and additive noise, respectively. For $\epsilon=0$ we have a purely multiplicative process, studied by many authors. $^{4-8}$ It can be identified with a zero-dimensional time-dependent Ginzburg-Landau model in which the coefficient of the linear terms fluctuates around a mean value $r$. A generalization to a $n$-component stochastic process as required for a laser system $(n=2)$ [Ref. 3(b)] or a chemical autocatalytic reaction ${ }^{14}$ is straightforward. The process $x(t)$ has a natural boundary at $x=0$ so that it is confined to a subspace of phase space in which either $x>0$ or $x<0$. The introduction of the additive noise $\xi(t)$ makes possible the connection between these two subspaces and restores the symmetry of the problem. We wish here to study the decay of an unstable state $x(t=0)=0$. Such decay is only possible for $\epsilon \neq 0$. Our analysis considers situations in which $\epsilon / r^{2}<<D / r<1$. This corresponds to a typical situation in which $\xi(t)$ stands for some internal or thermal noise and $\eta(t)$ is thought to be a weak noise originating from external fluctuations of the control parameter. In the case of pumping fluctuations in a dye laser it is generally accepted [Refs. $11(\mathrm{~d})-11(\mathrm{f})]$ that the external multiplicative noise cannot be approximated by a white noise. It rather has a finite correlation time. The same thing happens in other external noise problems (Ref. 5). In a first attempt to understand the role of multiplicative noise in the decay of an unstable state we take here a simple model and consider $\eta(t)$ a white noise for simplicity. An extension of our results is necessary for a detailed analysis of situations in which $\eta(t)$ is nonwhite. However, we do not foresee any drastic qualitative change in our general picture for $n=1$. In order to verify the main aspects of the decay process we have performed a numerical simulation of Eq. (1) in which we study the behavior of typical stochastic trajectories and also of statistical averages. We remark that in a system subject to external fluctuations what is observed in some circumstances is just the response of the system to a single realization of the external noise. This is for instance the case when the fluctuations of the control parameter are due to natural fluctuations of the environment of the system. Several experiments on the same system take the place of a single realization of the external noise. Stochastic trajectories are then directly observed. Results of the simulation are shown in Figs. $1-3$ for $r=1$ and $\epsilon=10^{-12}$ The simulation has been carried out with the algorithm explained in Ref. 8. The integration step was $\Delta=0.01$ and the averages are taken over $10^{4}$ stochastic trajectories.

In Fig. 1 three typical stochastic trajectories are shown. A comparison of results for different values of $D$ shows the different roles of the two sources of noise. One can distinguish three time regimes. The first one is up to the time in which the system leaves the unstable state. In the second regime the system decays to the stable state and in the third regime the system fluctuates around that stable state. The first regime is dominated by the additive noise. The extension of this regime depends slightly on $D$ (for moderate values of $D$ ) since the time at which the system leaves the unstable states decreases slightly with $D$. In the second regime the shape of the trajectory is largely independent of $\epsilon$ while the effect of $D$ becomes noticeable when the trajectory approaches the steady state. This effect is larger for larger $D$. In the final regime multiplicative fluctuations dominate. The overall behavior is easy to understand since for small $x$ the term with multiplicative fluctuations in (1) is damped out.

In Fig. 2 the transient mean value $\left\langle x^{2}(t)\right\rangle$ is shown. The main features discussed for the stochastic trajectories are also seen here, namely the existence of three time regimes in which additive noise and multiplicative noise play different roles. A slight decrease with increasing $D$ 


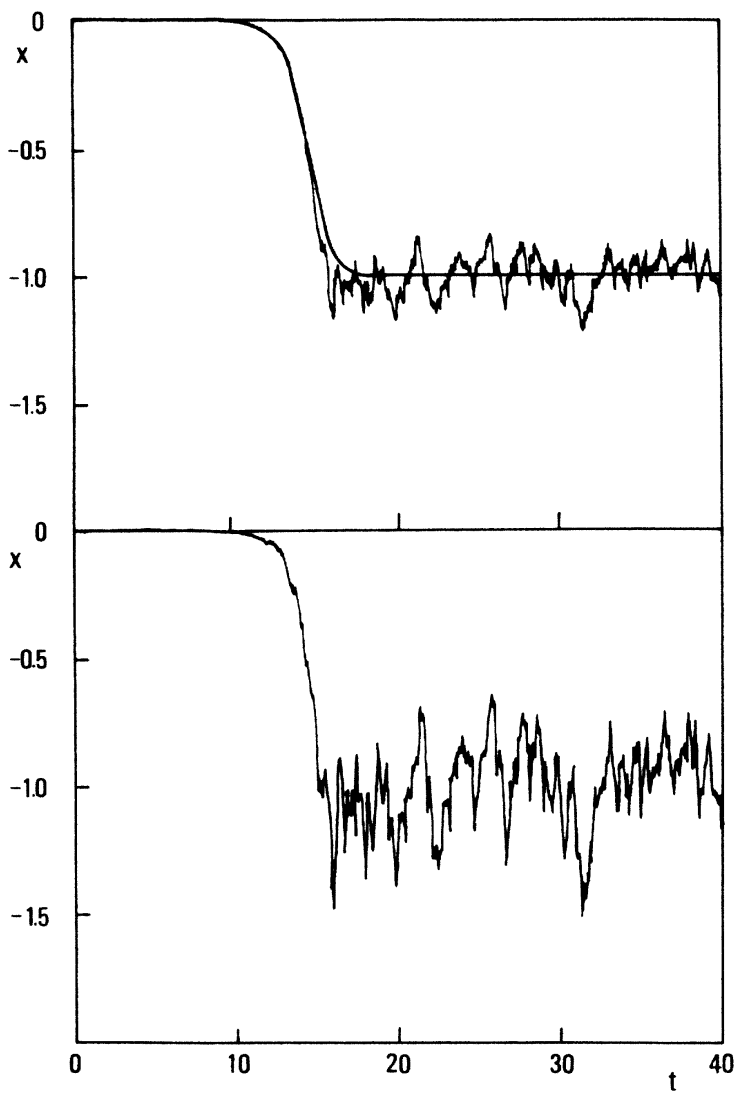

FIG. 1. Typical stochastic trajectories of the process $x(t)$ for $\epsilon=10^{-12}$ and $D=0$ and 0.01 (above) and for $D=0.05$ (below).

of the time at which the system leaves the unstable state and a slight growth of the time at which it reaches the final state are also seen. Figure 3 shows the phenomenon of anomalous fluctuations as modified by multiplicative noise. Again we observe here a separation of time scales, a preponderance of multiplicative noise at late times, and a slight decrease with increasing $D$ of the time at which fluctuations start to grow. A large increase with $D$ of the height of the peak of $\delta(t)=\left\langle x^{4}(t)\right\rangle-\left\langle x^{2}(t)\right\rangle^{2}$ appears as a new characteristic feature. The position of the peak remains essentially unchanged. The origin of the anomalous fluctuations for $D=0$ is known to be the spread of stochastic trajectories at intermediate times due to the random times at which they leave the unstable state. In the presence of multiplicative fluctuations there is an additional spread caused by multiplicative noise. This additional spread of trajectories due to multiplicative noise is also present in the final state and leads to a final value of $\delta$ larger than for $D=0$. The anomalous fluctuations $\Delta$ associated with the decay are given by the difference in the value of $\delta$ at its maximum and the asymptotic value for $t \rightarrow \infty, \Delta=\delta\left(t_{\max }\right)-\delta(\infty)$. Such anomalous fluctuations are essentially independent of the value of $D$. Multiplicative noise has an increasingly strong effect on the trajectories once the system is not close to the unstable state. The situation in which only multiplicative noise is present produces a monotonic growth of fluctuations until

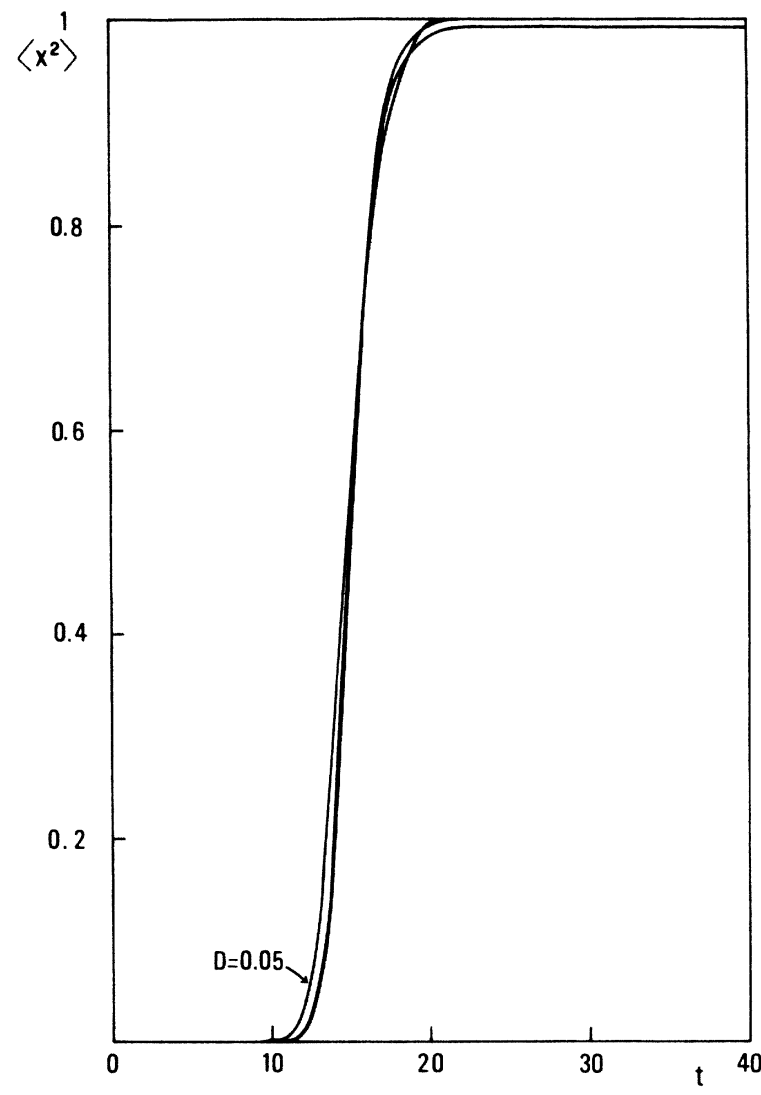

FIG. 2. Transient mean value $x^{2}(t)$ for $\epsilon=10^{-12}, D=0$, 0.01 , and 0.05 .

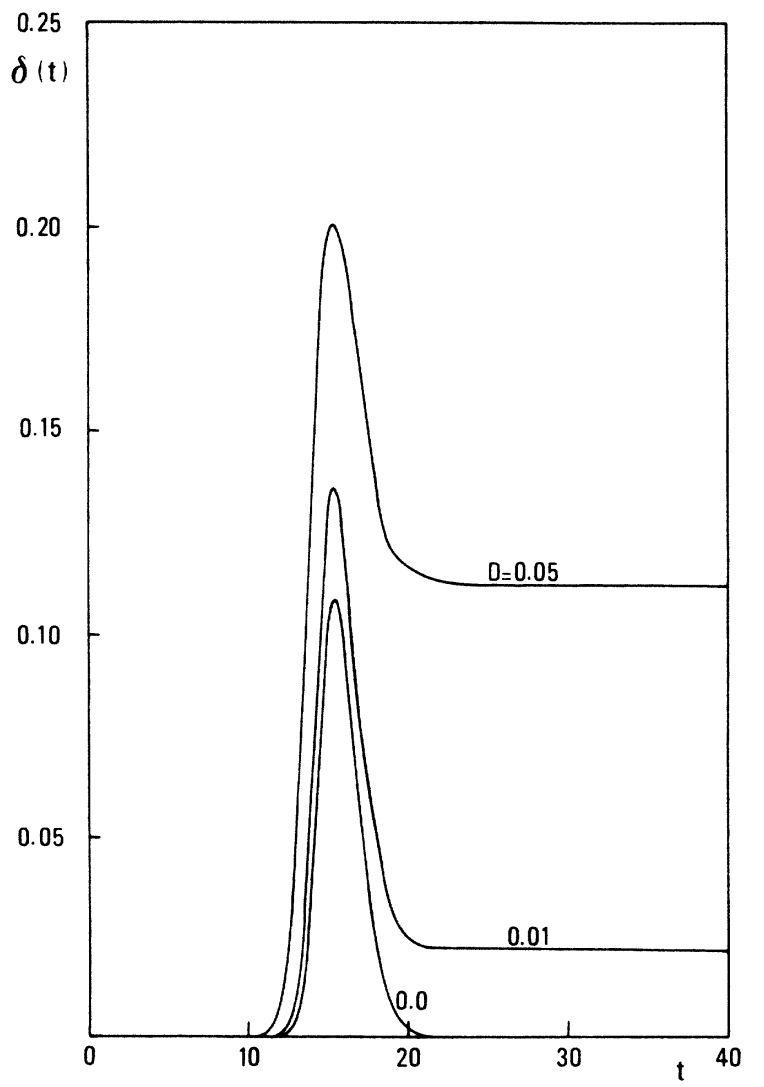

FIG. 3. Effect of multiplicative noise on the anomalous fluctuations. 
the maximum level is reached at the steady state. An enhancement of fluctuations above such a level is present if the spread of trajectories due to additive noise at intermediate times is larger than the typical spread of trajectories at the steady state. The first spread is of the order of $(r / 2)^{2}$ and the latter is of the order of $D^{2}$. Therefore, the typical phenomenon of transient fluctuation enhancement is only expected to be present if $D \leqslant r / 2$.

\section{THE QUASIDETERMINISTIC THEORY}

The numerical simulation of the process involving both additive and multiplicative noise discussed in Sec. II shows a clear separation of time scales, at least when the strength of the two types of noise is not very large. In other words, additive noise is the main cause for the decay of the unstable initial state, while multiplicative noise dominates the late-stage dynamics. Making a comparison with the case in which $D=0$, we see that the crucial role of additive noise for early times is the same. In that case, the QDT basically consists of replacing the true stochastic process by the deterministic solution of the problem $(\epsilon=0)$ with random initial conditions which account for the early-time fluctuations responsible for the decay of the unstable state. A QDT in the presence of multiplicative noise will be constructed in the pure multiplicative process. As a consequence of the picture obtained from the simulation we expect that such a QDT will describe reasonably well the temporal development of the system. For early times the essential additive fluctuations are taken into account. In the late regime multiplicative fluctuations dominate and the QDT leads by construction to the purely multiplicative process.

The first step of the QDT is to linearize the equation of motion close to the instability:

$$
\partial_{t} x=r x+\sqrt{2 \epsilon} \xi(t) \text {. }
$$

The linearized equation describes the early-time regime in which multiplicative fluctuations are neglected. The solution of (3) can be written as

$$
\begin{aligned}
& x(t)=h(t) e^{r t}, \\
& h(t)=\sqrt{2 \epsilon} \int_{0}^{t} d t^{\prime} e^{-r^{\prime}} \xi\left(t^{\prime}\right)
\end{aligned}
$$

and shows that the process $h(t)$ plays the role of a stochastic initial condition for the linearized motion. The process $h(t)$ has the important property of becoming a random variable $h_{\infty}$ independent of time for long times, as it can be easily seen from the stochastic differential equation

$$
\partial_{t} h=\sqrt{2 \epsilon} e^{-r t} \xi(t) .
$$

It is therefore clear that for times of the order of $1 / r$ the stochastic trajectories of the process (4) will coincide with the deterministic trajectories with initial condition $h_{\infty}$. From this time on, the system will evolve in a way which is essentially not influenced by additive noise. In the present case this means that the evolution equation is given by the purely multiplicative process

$$
\partial_{t} x=r x-u x^{3}+\sqrt{2 D} x \eta(t),
$$

which has the solution

$$
x(t)=\frac{x(0) e^{r t+w(t)}}{\left[1+2 u x^{2}(0) \int_{0}^{t} d t^{\prime} e^{2\left[t^{\prime}+w\left(t^{\prime}\right)\right]}\right]^{1 / 2}}
$$

with

$$
w(t)=\sqrt{2 D} \int_{0}^{t} d t^{\prime} \eta\left(t^{\prime}\right)
$$

the Wiener process and $x(0)$ the initial condition of the motion. In order to avoid the introduction of a connecting time between the initial linearized regime and the later one with $\epsilon=0$, the QDT makes the ansatz that the process will be well approximated by

$x_{\mathrm{QDT}}(t)=\frac{h(t) e^{r t+w(t)}}{\left[1+2 u h^{2}(t) \int_{0}^{t} d t^{\prime} e^{2\left[t^{\prime}+w\left(t^{\prime}\right)\right]}\right]^{1 / 2}}$,

i.e., by process (8) in which the initial condition has been substituted for all times by the process $h(t)$.

The process (10) gives the solution to the problem in the quasideterministic approximation. This solution reduces the original problem (1) to the purely multiplicative stochastic process (7) but with a random initial condition $h(t)$ distributed according to a time-dependent Gaussian with zero mean and variance $\left\langle h^{2}(t)\right\rangle=(\epsilon / r)\left(1-e^{-2 r t}\right)$. An exact evaluation for the statistical averages of the multiplicative process (7) is known. ${ }^{7}$ In particular, transient moments of that process are given in terms of the initial values $x(0)$. Our approximation to the original process is obtained by replacing $x(0)$ by $h(t)$. Moments of the process (10) can then be calculated exactly by a Gaussian averaging over the stochastic trajectories of the process $h(t)$. After times of order $1 / r$ this averaging is equivalent to an average with respect to an effective initial Gaussian distribution with variance $\epsilon$.

The validity of the QDT can be checked at two different levels. The first one is to consider directly the process (10) and its trajectories and compare them with the trajectories of the original process (1). Some examples of QDT trajectories are shown in Fig. 4 and can be directly compared with the corresponding ones of Fig. 1 since they refer to the same stochastic sequence in time. The overall agreement of the real and the stochastic trajectories is very good. The small deviation after the time in which the system has left the instability is due to the fact that we have ignored the effect of multiplicative noise on the initial stage of the motion and this has an effect for times around the mean first-passage time. This drawback is due to the fact that in order to have the simplest QDT process we do not describe correctly the linear regime. For later times it is quite evident that the dominant effect is due to the multiplicative noise which has the same effect on both the real and QDT trajectories. We should note at this point that QDT is unable to reproduce correctly the fluctuations in the final state due to the additive noise when $D=0$, since in that approximation the motion remains deterministic for long times. The situation is quite different for the multiplicative noise which is exactly taken into account when $\epsilon=0$. Since in our case we have a multiplicative noise much stronger than the additive one, the possible improvements of QDT [see Refs. 3(b) and 15] as 


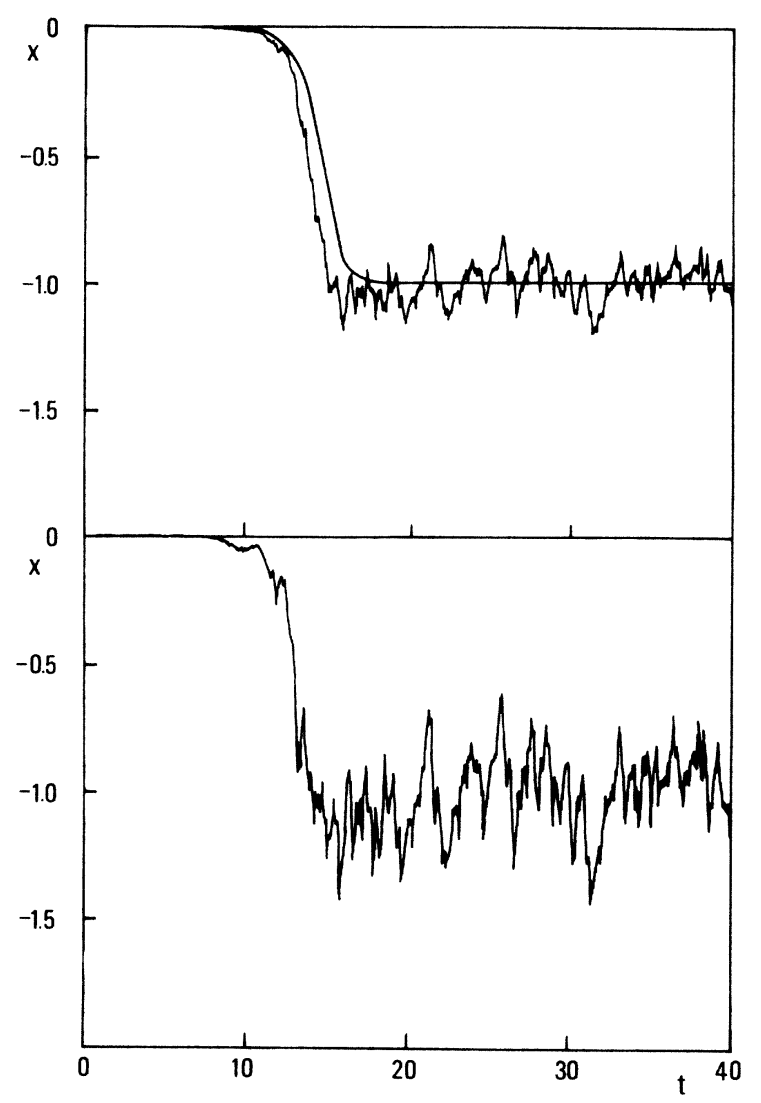

FIG. 4. Same trajectories as in Fig. 1 obtained with the QDT.

far as the additive noise is concerned are here irrelevant.

The other check of the theory can be made at the level of moments. Since we have a very good approximation for the trajectories of the process we expect a good agreement as far as averaged quantities are concerned. In fact, the agreement for the lowest-order moment $\left\langle x^{2}\right\rangle$ is excellent, and we use the variance of $\left\langle x^{2}\right\rangle$ in order to point out possible discrepancies. Figure 5 shows the anomalous fluctuations in the transient obtained with the QDT and for the same values of the parameters as in Fig. 3, the results of which are very well reproduced.

A general comment we can make about the moments of the original process and its QDT version is the loss of the scaling regime that was the characteristic feature of the problem with no multiplicative noise. In this case the moments could be written as a universal function ${ }^{1}$ of a single scaled time variable. It is, on the contrary, apparent from the expressions given in Ref. 7 that the same property is not valid when $D \neq 0$. On the other hand, the asymptotic properties in time of the moments calculated in Ref. 7 remain valid even after the Gaussian integration over the initial conditions implied by the introduction of the additive noise. In fact, the time scale of the decay [starting from $x(0) \neq 0$ ] when only the multiplicative noise is present is of the order of the inverse of the smallest eigenvalue $\tau_{1} \approx 2 r-4 D$. However, this is not the slowest relaxation time scale of the system. In fact, the presence of ad-

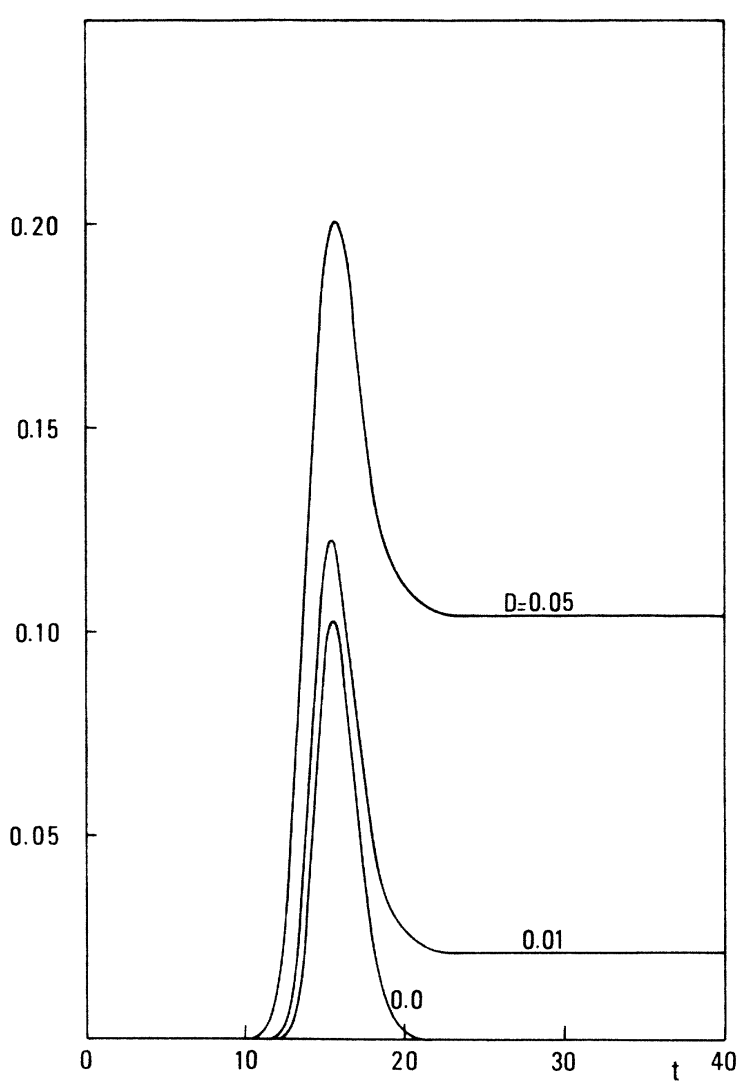

FIG. 5. Anomalous fluctuations in the QDT.

ditive noise will produce rare but large fluctuations which mix trajectories when they are close to the two equilibrium states. Such a final relaxation-time regime can be evaluated studying the mean first-passage time between the two potential wells in the presence of both multiplicative and additive noise. This study was done in Ref. 10 and gives a result valid for both $D$ and $\epsilon$ small in comparison with $r$ and $\epsilon<<D$,

$$
\tau_{2} \simeq \frac{\pi \sqrt{2}}{|r|}\left(-\frac{r D}{\epsilon}\right)^{-r / 2 D} e^{r / 2 D}
$$

a time much larger than $\tau_{1}$, so that the decay process calculated using the results of Ref. 7 will be observed for $t<<\tau_{2}$.

Since the calculation of the moments is quite cumbersome either when using the quasideterministic process or the results of Ref. 7, we try here to characterize in simple terms some of the main features of the moments themselves. Two such features are the facts that fluctuations start to grow at an earlier time than for $D=0$ and that the position of the maximum of $\delta(t)$ is essentially independent of $D$. If we use the QDT in the linearized regime it is easy to calculate the time $\tau_{0}$ the system takes to reach a given value of $\left\langle x^{2}(t)\right\rangle$, 


$$
\begin{aligned}
\tau_{0} & \approx \frac{1}{2 r} \ln \left(\frac{\left\langle x^{2}\right\rangle}{\epsilon}\right)\left(1-\frac{D}{r}\right) \\
& \simeq\left(\frac{1}{2 r} \ln \frac{1}{\epsilon}\right)\left(1-\frac{2 D}{r}\right),
\end{aligned}
$$

where $D / r \ll 1$ and $\ln \left\langle x^{2}\right\rangle \ll|\ln \epsilon|$. The displacement of the time it takes for the moments to become appreciably different from zero is therefore easily calculated from (12) and is in good agreement with the simulation. We may also note that $T_{0} \approx(1 / 2 r) \ln (1 / \epsilon)$ corresponds to the mean first-passage time to leave the instability when only additive noise is present. One might therefore think that what we calculated above is the correction to the mean first-passage time when we also have multiplicative noise. However, if we try to evaluate the mean firstpassage time $T$ we get, in the linear QDT regime,

$$
\left[T-\frac{1}{2 r} \ln \frac{x^{2}}{h^{2}(T)}\right]^{2}=\frac{w^{2}(T)}{r^{2}} .
$$

This equation should be solved in $T$ for a fixed $x^{2}$ and averaged over the multiplicative and additive noise. An order of magnitude of $T$ can be obtained using for the processes $h^{2}(t)$ and $w^{2}(t)$ the estimates $\left\langle h^{2}(T)\right\rangle \approx \epsilon$ and $\left\langle w^{2}(T)\right\rangle=2 D T$. This leads, for small values of $D / r^{2}$, to

$$
T \approx T_{0} \pm \frac{\sqrt{2 D T_{0}}}{r}
$$

Equation (14) limits the possible shift in $T_{0}$ to a very small value compared to the one obtained above, for instance for $D=0.01$ and $\epsilon=10^{-12}$. Our conclusion is therefore that the displacement in time of the moments for small times does not reflect a variation of the mean first-passage time due to multiplicative noise. At this point, we observe that the curve of the variance $\delta$ of $x^{2}$ does not shift as a whole by a quantity of the order of $T_{0} D / r$. Instead, the instant of time corresponding to the maximum fluctuations remains essentially fixed. We are therefore lead to the conclusion that this maximum is related to the mean first-passage time. This fact is, on the other hand, confirmed by the observation that the time when the spread of trajectories is maximum basically depends on the additive noise and it is modified by multiplicative noise much less than the magnitude of fluctuations.

\section{DISCUSSION AND COMMENTS}

We wish to comment here on the approximations involved in the QDT. In the case in which only additive noise is present, the linear version of QDT coincides with the linear version of the real process. This is not the case in the QDT approximation presented here when $D \neq 0$. From Eqs. (1) and (10) the two linear processes are given, respectively, by

$$
\begin{aligned}
& x^{l}(t)=\int_{0}^{t} e^{r\left(t-t^{\prime}\right)+w\left(t-t^{\prime}\right)} \sqrt{2 \epsilon} \xi\left(t^{\prime}\right) d t^{\prime}, \\
& x_{\mathrm{QDT}}^{l}(t)=e^{w(t)} \int_{0}^{t} e^{r\left(t-t^{\prime}\right)} \sqrt{2 \epsilon} \xi\left(t^{\prime}\right) d t^{\prime} .
\end{aligned}
$$

The difference between these two processes can be evaluated looking for the relative standard deviation

$$
\sigma=\left(\frac{\left\langle\left(x^{l}-x_{\mathrm{QDT}}^{l}\right)^{2}\right\rangle}{\left\langle x^{l 2}\right\rangle}\right)^{1 / 2}
$$

For $D<r$ and $r t \gg 1$ we have

$\sigma=\left[\frac{4 D}{r}\left[1+\frac{2 D}{r}\right)\right]^{1 / 2}=\left(\frac{4 D}{r}\right)^{1 / 2}+O\left(\frac{D}{r}\right)$.

This result explains, for the values of parameters used here, the agreement found between the simulation and QDT on the first stage of evolution. The validity of QDT for long times only requires having $D r \gg \epsilon$. QDT is much better in this regime than in the case of $D=0$ since now additive noise plays no important role. Equation (18) restricts the validity of our theoretical description for short times to relatively small values of $D$. We recall that for intermediate times we also estimated in Sec. II that for $D \geq r / 2$ the phenomenon of anomalous fluctuations is hidden by large multiplicative noise. In fact, for large values of $D$ the system is dominated by noise, and the ordinary concepts of unstable states and the decay to stable states lose their usual physical meaning.

Our overall description of the decay process for $\epsilon / r^{2}<-D / r<1$ indicates a well-separated role of additive and multiplicative noise, namely, anomalous fluctuations and the position of the peak of $\delta(t)$ are fixed by the parameter $\epsilon$, the time at which fluctuations start to grow depends on $D$, and the final fluctuations are given by $D$. Therefore, transient experiments should be useful to determine the value of these two parameters.

We finally summarize the role of a small additive noise on a bistable system with a fluctuating control parameter. Its main effect is to mix trajectories which otherwise would remain confined in two separate subspaces of phase space close to the relative local minima $x= \pm(r)^{1 / 2}$. Such a mixing is verified by looking at the decay of an unstable state. This is well described by QDT. A related effect of additive noise can appear after the initial decay of the unstable state if multiplicative noise is large enough. In that case the system might often be brought close to the unstable state and a small additive noise can overcome the barrier separating the two locally stable states. This produces again the mixing of phase space in times which are much smaller than the characteristic times of barrier crossing in the case $D=0$. Such an equilibration process between the two locally stable states is not described by the QDT since the process $h(t)$, which contains the effect of additive noise, is saturated for long times. The equilibration occurs in a time scale given by the mean first-passage time to escape from one stable state to the other. We have seen in Sec. III that for relatively small $D$ this final dynamic regime is well separated from the relaxation regime described by the QDT. In a nonlinear relaxation experiment we expect that for observation times shorter than the escape time, the system will be observed in a metastablelike state. It is interesting to consider how the system will leave such a state. According to the study of Ref. 10, the escape time shows for small $\epsilon$ a quite sharp change of behavior with $D$, namely, it is very large for small $D$ and quite sharply decreases to a small value when $D \geq D_{c}$. A small $\epsilon$ permits for $D \geq D_{c}$ a rapid mixing of 
phase space. This means that in a given time scale of observation, $x$ will have a value different from zero for $D \leq D_{c}$ and it will vanish on the average for $D \geq D_{c}$. Although there is no clear determination of the value $D_{c}$, this phenomenon indicates an effective modification of the phase diagram of dynamical origin for a finite observation time. The phenomenon is reminiscent of what happens when the control parameter $r$ in (1) is not a random quantity but a periodic function of time. ${ }^{16}$

\section{ACKNOWLEDGMENTS}

This work has been supported by a NATO Grant. Two of us (J.M.S. and M.S.M.) also acknowledge financial support from the Comisión Asesora de Investigación Cientifica y Técnica (Spain) (CAICyT).
${ }^{1}$ M. Suzuki, in Order and Fluctuations in Equilibrium and Nonequilibrium Statistical Mechanics, edited by G. Nicolis, G. Dewel, and J. Turner (Wiley, New York, 1980).

${ }^{2}$ F. T. Arecchi, V. Degiorgio, and B. Querzola, Phys. Rev. Lett. 19, 1168 (1967); Phys. Rev. A 3, 1108 (1971); D. Meltzer and L. Mandel, Phys. Rev. Lett. 25, 1151 (1970).

${ }^{3}$ (a) F. de Pasquale and P. Tombesi, Phys. Lett. 72A, 7 (1979); (b) F. de Pasquale, P. Tartaglia, and P. Tombesi, Phys. Rev. A 25, 466 (1982).

${ }^{4}$ R. L. Stratonovich, Topics in the Theory of Random Noise (Gordon and Breach, New York, 1967), Vol. II.

${ }^{5} \mathrm{~W}$. Horsthemke and R. Lefever, Noise Induced Transitions (Springer, New York, 1983).

${ }^{6}$ A. Schenzle and H. Brand, Phys. Rev. A 20, 1628 (1979); R. Graham and A. Schenzle, Phys. Rev. A 25, 1731 (1982).

${ }^{7}$ R. Graham and A. Schenzle, Phys. Rev. A 26, 1676 (1982).

${ }^{8}$ J. M. Sancho, M. San Miguel, S. Katz, and J. D. Gunton, Phys. Rev. A 26, 1589 (1982).

${ }^{9}$ See, for example, R. Zwanzig, J. Stat. Phys. 9, 215 (1973), or the derivation of stochastic models for optical bistable sys- tems [R. Bonifacio, M. Gronchi, and L. A. Lugiato, Phys. Rev. A 18, 2266 (1981)].

${ }^{10}$ E. Guardia and M. San Miguel, Phys. Lett. 109A, 9 (1985).

${ }^{11}$ (a) K. Kaminishi, R. Roy, R. Short, and L. Mandel, Phys. Rev. A 24, 370 (1981); (b) R. Graham, N. Höhnerbach, and A. Schenzle, Phys. Rev. Lett. 48, 1396 (1982); (c) R. Short, L. Mandel, and R. Roy, ibid. 49, 647 (1982); (d) S. N. Dixit and P. S. Sahni, ibid. 50, 1273 (1983); (e) A. Schenzle and R. Graham, Phys. Lett. 98A, 319 (1983); (f) A. Hernández-Machado, M. San Miguel, and S. Katz, Phys. Rev. A 31, 2362 (1985).

${ }^{12}$ P. Lett, R. Short, and L. Mandel, Phys. Rev. Lett. 52, 341 (1984).

${ }^{13}$ Equation (1) is interpreted in the Stratonovich sense.

${ }^{14} \mathrm{~F}$. de Pasquale, P. Tartaglia, and P. Tombesi, Nuovo Cimento 69B, 228 (1982).

${ }^{15}$ F. de Pasquale, P. Tartaglia, and P. Tombesi, Z. Phys. B 43, 353 (1981).

${ }^{16}$ F. de Pasquale, Z. Racz, M. San Miguel, and P. Tartaglia, Phys. Rev. B 30, 5228 (1984). 